\title{
The Investigation of Electron-Optical Parameters Using Artificial Neural Networks
}

\author{
A.H. ISIK \\ Mehmet Akif Ersoy University, Department of Computer Engineering, Burdur, Turkey
}

\begin{abstract}
The optimization of scientific instruments is crucially important to increase the quality of measurements. A major challenge for the development of these experimental tools is the precise determination of focal parameters. Therefore, usage of an innovative technique that meets our requirements is desirable. Among intelligent algorithms, artificial neural network (ANN) has an advantage of obtaining the optical parameters data with high accuracy. One of the most popular geometries used in electrostatic optical devices is geometry with cylinder lenses. In this study, the artificial neural network is applied for the first time to the subject of the magnification parameters of threeelement electrostatic cylinder lenses for a wide range of values of the applied voltages. ANN-based optimization has been performed using Matlab/Simulink, and the performance analysis has also been conducted. High-performance results have been achieved using ANN approach. The commercial simulation package SIMION software is used as a data source for artificial neural network results. This approach provides new perspectives for the effective solution for the problems related to electrostatic lenses with different geometries.
\end{abstract}

DOI: $10.12693 /$ APhysPolA.127.1317

PACS: 41.85.Ne, 07.05.Rm, 84.35.+I, 07.05.Mh

\section{Introduction}

Today's intelligent control systems have drawn a significant interest in many fields of physics. Surveys of these intelligent systems have been conducted in nuclear physics [1-3], mathematical physics [4], medical physics [5] and optical physics [6]. In addition, intelligent systems can be applied to electrostatic lenses. A charged particle beam focused with the aid of electrostatic lenses is has important applications in many experimental designs. Therefore, the optimum lens designs have been extensively studied theoretically using computer-based techniques [7-9]. As in most areas, applications of intelligent systems to optimize the lens design have gained popularity in recent years [10-12]. The aim of this investigation is to find the focusing condition of electrostatic lenses by using artificial neural networks (ANNs). In this study, the artificial neural network is employed for the first time to obtain electron optical parameters of three-element cylindrical lenses. The artificial neural network algorithm requires a set of known input-output parameters, used to train and test the neural network. The electron ray-tracing simulation package SIMION software is used as a data source for the neural network. It is also employed for validation of network results [13]. This study focuses on the three-element electrostatic zoom-lenses for a wide range of the applied voltage values $\left(V_{3} / V_{1}\right.$ and $\left.V_{2} / V_{1}\right)$. The proposed network classifies two modes of three-element lenses: focused and unfocused condition. This method enables the determination of the unknown focusing condition by using known

e-mail: ahakan@mehmetakif.edu.tr data with high-accuracy. It provides the time advantage for the optimization of experimental lens designs.

This study is organized as follows. In Section 2, the information of focal parameters for three-element lenses and the fundamentals of neural networks are briefly reviewed. In Section 3, the performance analysis results of ANN algorithm are presented. Finally, conclusion and recommendation of the issue of investigation of electronoptical parameters using artificial neural networks are given in Section 4.

\section{Materials and method}

The focal parameters of a lens system are basically described with the first and second focal points $\left(F_{1}\right.$ and $\left.F_{2}\right)$, the object distance $(P)$, and image distance $(Q)$. At fixed image and object position, at least three-element lens can maintain the magnification parameter. In this condition, three-element lens systems can be used in experimental tools as zoom lenses. A three-element electrostatic lens with the diameter $(D)$ is shown in Fig. 1. " $A$ " shows the length of the center lens with the half of the gap to each side. The potentials $V_{i}$ stand for the applied lens voltages. $P$ and $Q$ are the object and image distances from the center of the lens system, respectively.

In Figure 1, the electron trajectory in image plane $(Q)$ has a beam diameter $(\Delta r)$, where the charged particles are influenced by aberrations and distortions. Thus, determination of the lens systems, used for focusing of a beam of charged particles, requires the knowledge of the calculation results of the potential function $\left(V_{r}\right)$ throughout the trajectory of particles. Up to now, the focal parameters of lenses have been obtained by various calculation methods. Some of the commonly used methods for the solution of partial differential equation, are the separation variables method [14], the boundary element (BEM) [15], the finite element method [16], the finite 
difference method [17]. These methods have been used to solve Laplace equation. In all of the above methods, the SIMION program uses the finite-difference method by means of the nine-point relaxation techniques [13]. In present work, the feed-forward artificial neural network is trained and tested using the SIMION data.

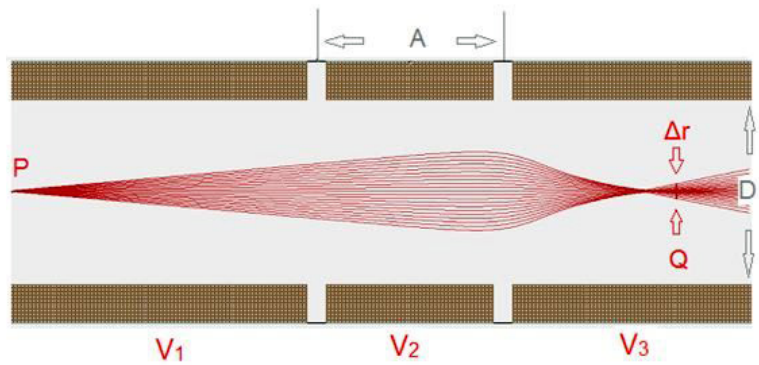

Fig. 1. A schematic view of three-element cylindrical lens system.

Artificial neural network approaches are generally used for control of non-linear, complex, time-varying systems. The artificial neural networks are based on a learning of input-output logical behavior. Basically, ANNs are modeled on the principles of behavior of human brain. The neurons are principal elements in these networks, just as neuronal cell. Signals are delivered from the inside to outside of biological cells by means of dendrites. If incoming signal is bigger than the cell threshold, it is transmitted to axon. The mathematical modeling of this nervous system is the artificial neural network [18]. Artificial neurons have been arranged in multi-layers consisting of input, hidden and output layers. Each neuron from input layer is connected to a neuron from the hidden layer. Output layer processes data from the hidden layer and generates an output.

In this study, feed-forward back propagation algorithm [19] is used for the ANN architecture. The proposed neural network is schematically represented in Fig. 2. The input layer consists of two neurons (voltage ratios; $V_{3} / V_{1}$ and $\left.V_{2} / V_{1}\right)$. Similarly, the output layer has two neurons, focused and unfocused condition. In this algorithm, all information for training is given and network tunes the weights for optimum ANN outputs. The sigmoid activation function $\left(1 /\left(1+\mathrm{e}^{-x}\right)\right)$ is used both in hidden and output layers. In the training stage, backpropagation algorithm with Levenberg-Marquardt [20] is used. When the ANN training process reaches to a minimum error, it is tested with the unknown data.

The flowchart of classification of optical parameters using ANN is shown in Fig. 3. Firstly, the feature selection is performed within various input and output values. Then, the ANN architectures with different hidden layers and activation functions are designed. After training and testing of ANN with the data of SIMION software, performance analysis through accuracy, sensitivity, specificity, and MSE (Mean squared error) are done. Depending on these results, classification process is terminated or new ANN architecture is defined to obtain best classification results.

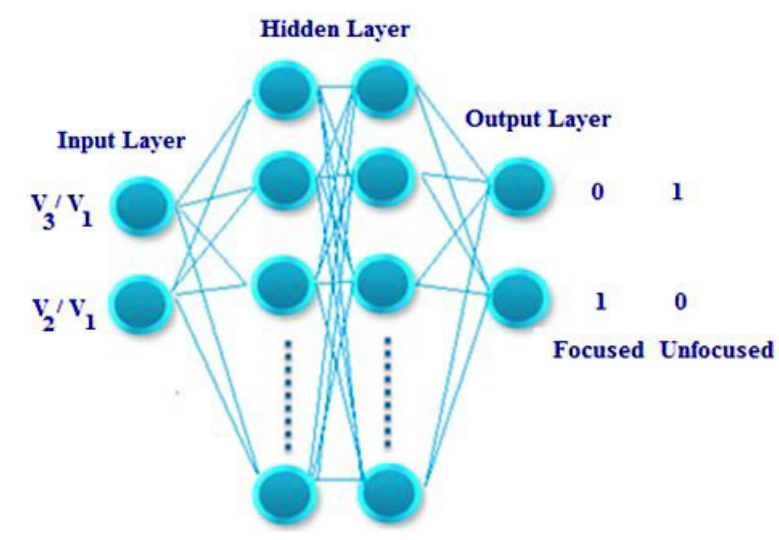

Fig. 2. The ANN schematic topology.

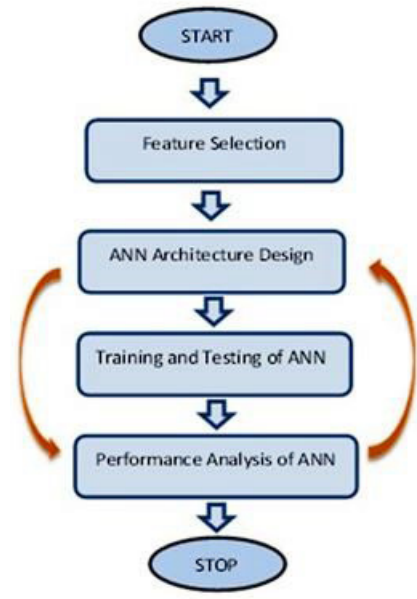

Fig. 3. Flowchart of classification of optical parameters using ANN.

\section{Results}

In the present work, the feed-forward back propagation learning algorithm has been used for the determination of focusing condition of three-element cylindrical lenses, using fixed values of object $(P / D=5)$ and image distance $(Q / D=5)$. Neural network toolbox in MATLAB/Simulink software package has been used in the study. To improve performance results, a normalization technique is applied to input values. Input and output data of ANN obtained from SIMION software are divided into $70 \%$ training set, $15 \%$ validation set and $15 \%$ test set, consisting of 77,16 , and 16 samples. The performance analysis of neural network is evaluated by means of accuracy, sensitivity, specificity, MSE and ROC (Receiver operating characteristic) curve [18]. According to the test results, the 2-10-2 ANN architecture has the best results in classifying of optical parameters. Test results of this architecture have highest performance values with $100 \%$ accuracy, $100 \%$ sensitivity, $100 \%$ specificity, and 0.030 MSE respectively. All performance results of training and testing process for different ANN architecture are given in Table I.

In 2-10-2 architecture, all unknown samples are classi- 
Performance results of different ANN TABLE I architecture.

\begin{tabular}{c|c|c|c|c|c|c|c|c}
\hline \hline \multirow{2}{*}{$\begin{array}{c}\text { Archi- } \\
\text { tecture }\end{array}$} & \multicolumn{4}{|c|}{ Training Results } & \multicolumn{4}{c}{ Test Results } \\
\cline { 2 - 9 } & $\begin{array}{c}\text { racu } \\
\text { racu- }\end{array}$ & $\begin{array}{c}\text { Sensi- } \\
\text { tivity }\end{array}$ & $\begin{array}{c}\text { Speci- } \\
\text { ficity }\end{array}$ & MSE & $\begin{array}{c}\text { Accu- } \\
\text { racy }\end{array}$ & $\begin{array}{c}\text { Sensi- } \\
\text { tivity }\end{array}$ & $\begin{array}{c}\text { Speci- } \\
\text { ficity }\end{array}$ & MSE \\
\hline $2-4-2$ & 85.7 & 96.9 & 77.8 & 0.116 & 93.8 & 100 & 90.9 & 0.069 \\
\hline $2-6-2$ & 88.8 & 96.9 & 93.3 & 0.062 & 87.5 & 100 & 77.8 & 0.121 \\
\hline $2-8-2$ & 94.8 & 100 & 90.9 & 0.054 & 93.8 & 100 & 90 & 0.071 \\
\hline $2-9-2$ & 93.5 & 100 & 88.6 & 0.044 & 93.8 & 100 & 90 & 0.053 \\
\hline $2-10-2$ & 94.2 & 100 & 95 & 0.075 & 100 & 100 & 100 & 0.030 \\
\hline $2-12-2$ & 87 & 100 & 76.2 & 0.091 & 93.8 & 100 & 91.7 & 0.065
\end{tabular}

fied correctly. It is also understood from the $100 \%$ sensitivity that designed neural network has the highest capability to identify those with the focused condition. Similarly, 100\% specificity means the network has the highest capability to identify those without the focused condition.

ROC analysis is also applied to measure classification ability of ANN. ROC analysis results for 2-10-2 ANN architecture is given in Fig. 4. Obtained ROC curve draws the true-positives (sensitivity) versus false-positives (1specificity). Areas below the class 1 (focused condition) and 2 lines (unfocused condition) demonstrate the best predictive ability. The area under the test ROC curve is 1. It represents a perfect classification result.
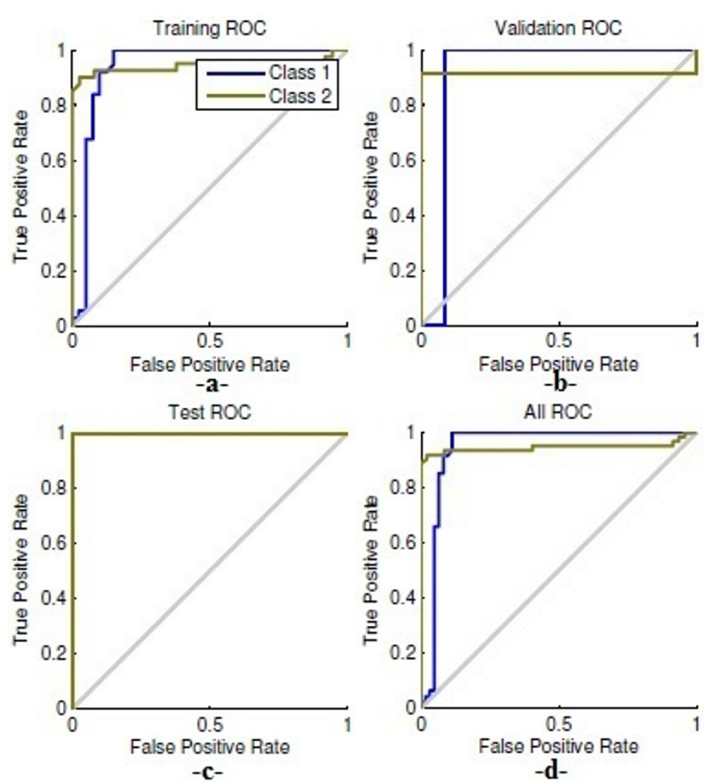

Fig. 4. ROC curve results of 2-10-2 ANN for (a) training dataset (b) validation dataset (c) test dataset (d) all dataset.

\section{Conclusion}

In this study, the novel approach is performed to obtain the zoom-lens properties of three-element cylindrical electrostatic lenses. Beam diameter data in image plane train the artificial neural network reasonably well and the appropriate weights tune to obtain focusing condition of lens system. Using a different number of hidden layers, the network is evaluated to achieve best performance with minimum error. The obtained 2-10-2 ANN results are found to be in good agreement with the original simulation data. This ANN approach is a perfect method to determine the focusing condition. The results show that the artificial neural networks are capable to identify the optical property of electron beams in threeelement zoom lenses with high-performance values. The network permits rapid solution (in a few seconds) to tune optimum lens voltages for desirable lens condition. In the future, this artificial neural networks method can be used for different types of lens system, as data analysis tool.

\section{Acknowledgments}

I would like to thank SIMION researcher for delivering a test data for checking our results.

\section{References}

[1] K.L. Peterson, Phys. Rev. A 41, 2457 (1990).

[2] W.L. Morgan, IEEE Trans. Plasma Sci. 19, 250 (1991).

[3] K.A. El-Metwally, T.I. Haweel, M.Y. El-Bakry, Int. J. Mod. Phys. C 11, 619 (2000).

[4] T. Nguyen-Thien, T. Tran-Cong, App. Math. Model. 23, 687 (1999).

[5] J. Khan, J.S. Wei, M. Ringner, L.H. Saal, M. Ladanyi, F. Westermann, F. Berthold, M. Schwab, C.R. Antonescu, C. Peterson, P.S. Meltzer, Nature Medicine 7, 673 (2001).

[6] D. Petkovic, N.T. Pavlovic, S. Shamshirband, M.L. Mat Kiah, N. Badrul Anuar, M.Y. Idna Idris, Opt. Lasers Eng. 55, 84 (2014).

[7] D.W.O. Heddle, Electrostatic Lens Systems, IOP Press, London 2000.

[8] O. Sise, M. Ulu, M. Dogan, Nucl. Instr. Meth. A. 554, 114 (2005).

[9] O. Sise, N. Okumus, M. Ulu, M. Dogan, J. Elect. Spectr. Rel. Phen. 175, 76 (2009).

[10] S.C. Johnston, W.A. Greynolds, D.Y. Wang, D.C. Dilworth, Proc. SPIE 1780, 192 (1993).

[11] S.M. Tam, C.K. Kwong, W.H. Ip, Int. J. Comp. Appl. Techn. 13, 229 (2000).

[11] A.A. Fadhil, Int. J. Adv. Comp. Tech. 2, 66 (2010).

[12] Simion 3D v8.1, Scientific Instrument Services Inc., http: //www.simion. com, 2012.

[13] A.B. El-Kareh, J.C.J. El-Kareh, Electron Beams, Lenses and Optics 1-2, Academic Press, London 1970.

[14] A. Renau, F.H. Read, J.N.H. Brunt, J. Phys. E: Sci. Instrum. 15, 347 (1982).

[15] D.W.O. Heddle, J. Phys. D: Appl. Phys. 32, 1447 (1999).

[16] A. Khursheed, The Finite Element Method in Charged Particle Optics, Kluwer, London 1999.

[17] I.A. Galushkin, Neural networks theory, Springer, Berlin 2007.

[18] A.H. Isik, İ. Güler, M.U. Sener, Telemed E. Health 19, 24 (2013).

[19] D. Marquardt, SIAM J. Soc. Ind. Appl. Math. 11, 431 (1963). 